\title{
Listening for Values
}

\author{
Mary C. Gentile ${ }^{1}$
}

Received: 4 April 2016/Accepted: 25 May 2016/Published online: 22 July 2016

(C) Springer International Publishing 2016

\begin{abstract}
Giving Voice To Values (GVV) is an innovative approach to values-driven leadership development and ethical practice that is being introduced around the world in business practice, business education and beyond. As GVV expands, one of its core concepts - the efficacy of "rehearsal" for values-driven action and voice - is also being used to build the muscle and predilection for skillful "listening" to values issues. This essay describes this experience in several organizations.
\end{abstract}

Keywords Giving Voice To Values · GVV · Values · Business ethics · Leadership · Leadership development $\cdot$ Pedagogy $\cdot$ Curriculum

\section{What is Giving Voice to Values?}

Over the past 10 years or so, I have been developing, refining, expanding and sharing an innovative approach to values-driven leadership development titled "Giving Voice To Values" (GVV). GVV is not about persuading people to be more ethical. Rather GVV starts from the premise that many of us already want to act on our values, but that we also want to feel that we have a reasonable chance of doing so effectively and successfully. This pedagogy and curriculum are about raising those odds.

Rather than a focus on ethical analysis, the Giving Voice to Values pedagogy focuses on ethical implementation and asks the question: "What if I were going to act on my values? What would I say and do? How could I be most effective?" Drawing on actual experience as well as scholarship, GVV fills a long-standing and critical gap in the development of values-centered leaders.

Originally developed for use in graduate business education, GVV has extended beyond that initial audience. Currently it has enjoyed well over 890 pilots on all seven continents and growing, and it has been used in MBA programs, undergraduate business programs, and executive education. Its use in corporations around the world has also grown, and although

Mary C. Gentile

GentileM@darden.virginia.edu; http://www.GivingVoiceToValuesTheBook.com

1 University of Virginia Darden School of Business, Charlottesville, VA 22903, USA 
often adopted by the Corporate Compliance practitioners, increasingly it is the Leadership Development professionals who have expressed interest in GVV. And finally, it has begun to be piloted in legal education, engineering education, nursing education, the military, public sector, and so on.

\section{The Link Between Voicing and Listening for Values}

But despite the rapid growth of Giving Voice To Values around the world - or perhaps because of it - I have occasionally seen an interesting misunderstanding of the approach that can sometimes surface. The very appeal of GVV - its aspirational nature, the hope that we might be able to actually act in alignment with our values and do so effectively - can sometimes trigger a somewhat closed-minded or even contentious stance that, ironically, is precisely the opposite of the stance GVV is intended to foster. And sometimes GVV is even misinterpreted as an approach to "whistle-blowing", when in fact, GVV was developed partly because whistle-blowing is so often ineffective or at best, a very costly way (for all parties) to address values conflicts. And it is often akin to closing the barn door after the horses have escaped. GVV is intended, instead, to help individuals develop the skills and scripts and comfort and predilection to action that is needed to effectively raise values conflicts before whistle-blowing becomes necessary or becomes the only way to proceed.

GVV is focused on preparing individuals for values-driven Voice and Action, as opposed to focusing exclusively on teaching the rules and frameworks needed for the Analysis of values conflicts (an important topic but one that is too often the sole focus of ethical training), and it is indeed this emphasis upon effective action that requires a more open, less contentious positioning for successful values-based communication.

Which leads me to the focus of this essay: that is, Listening for Values. Now to be honest, listening for values has been a key component of GVV from the very start. The very protocol of questions and analysis that lie at the heart of the GVV process for crafting effective scripts and action plans requires us to ask and answer the query: "What is at stake, or at risk, for all affected parties with regard to the values conflict at hand?" We ask this question not as part of a traditional stakeholder analysis; indeed, the GVV protocol is post-decision-making and as such, a stakeholder analysis would have already been completed prior to the crafting of scripts and action plans.

Rather we ask this question in order to understand what the key concerns, fears, aspirations, objectives may be for the very individuals whom we wish to influence or persuade. We want to understand if there are ways we can mitigate their concerns and fears, or at the least, acknowledge and respect them. And we want to find alternate ways to achieve their objectives, if appropriate; and if their objectives appear to be inappropriate or unethical at their core, we want to attempt to proffer compelling alternatives for them.

\section{The Question}

So the question becomes: how can we harness the aspirational motivation that derives from the desire to voice our values, without closing off our capacity and commitment to listening for values in others? As I travel around, sharing GVV, I will sometimes encounter individuals who say something like the following: "Well, I have never been afraid or reluctant to voice my values. However, even when I do, other people don't change." This statement is often 
delivered in an odd emotional stew of righteous satisfaction seasoned with an angry sense of futility.

But I think it is important to consider what our true purpose is when we voice our values. That is, do we want at the end of the day to simply be able to say "I expressed my views!" and leave it at that? Or do we want to be able to actually make change, for the good, in our organizations?

\section{GVV at the TOP}

A possible remedy for this misinterpretation of GVV - that is, the focus on voicing values without enough attention to listening for them - emerged when we began to consider ways to share the Giving Voice To Values methodology with more senior organizational leaders.

As more and more companies have expressed interest in experimenting with GVV, new advantages and lessons have emerged from their experiences. For example, although organizations often begin their GVV journey with the decision to apply this approach to their training initiatives for middle and lower level employees, as they become more familiar with the concepts they often begin to see the relevance for more senior executives. The framing of GVV is slightly different for senior level executives, however. We have identified three ways to adapt GVV for organizational leadership.

First, while programs for middle and lower level employees often focus on preparing them to manage and voice values to those who are more senior to them (i.e., speaking up a level to their boss), GVV for upper level managers and executives is often focused on finding ways to enact and voice values to one's senior peers (i.e., speaking across the organization). These peers are individuals with significant influence and power and with whom maintaining a collegial and collaborative relationship is essential, and the most effective approach may be quite different- less formal, more relationship-focused, and so on.

Another way that GVV is adapted for more senior managers is to focus on the identification of approaches that managers can use to create and maintain an organizational culture that encourages and enables and supports voicing values.

But perhaps the most well-received adaptation of GVV for senior leaders is the one that helps us to address the question above regarding the relationship between voicing values and listening for them. That is, GVV can be adapted to provide opportunities for managers to prescript, rehearse and peer coach effective ways to hear and respond to the efforts of other (often lower level) employees when they raise values issues - that is, to listen for values. If and when employees do raise values-related questions, the manner in which their manager receives the message sends powerful signals about whether this sort of behavior is genuinely welcome.

For example, one organization invited managers to identify examples of times when their reports appropriately and effectively raised values conflicts with them (without using names), so as to generate a list of tactics that make it easier for these managers to hear these messages. By so doing, the company is encouraging these managers to reflect upon ways that they have heard these messages - whether and why and how they responded well - and it is also identifying a list of proven tactics that can be shared in their training with more junior employees. And these tactics will have greater impact and credibility because they were identified as appropriate and welcome by more senior managers in the same firm.

Recently I worked with an organization where the senior leadership and a group of middle managers participated in the same discussion about voicing values. When it came to the 
discussion of some GVV scenarios, the leadership team worked at their tables on answering the question: "How can managers raise this issue with me in a way that makes it easier for me to respond effectively?" Meanwhile the middle managers worked on the question: "How can I raise this issue effectively with my seniors in the organization-and what would I hope for from them in response?"

When the two groups came back together to debrief, it was interesting to see that most of the requests from the senior leaders had less to do with the content of the values issue and more to do with the manner in which it is raised. For example:" Present the information clearly and concisely so that I do not have to spend extra time simply to understand your concern (important because often values questions already require time commitments that has not been anticipated so it is helpful to try to mitigate that demand as much as possible). Present the information in a way that respects my own time pressures and potential challenges. Avoid accusations; give me the benefit of the doubt (important because the emotional content of this discussion can be high). Provide as much of the background data and analysis as possible, so that I will be better able to understand and vet the concern, again as a demonstration of your respect for my time constraints. Help me understand what sorts of action you recommend; even if I do not agree with your suggestions, this will help me to think about "next steps." Understand that I will need to do my own due diligence and cannot necessarily respond immediately with a "yes" or "no" answer; this does not mean that I am not taking you seriously."

Although many of these requests are just good communication strategies, it is often the case that when it comes to values-related issues, the emotions of the individual presenting the concern will sometimes lead them to seem more antagonistic, more accusatory and more contentious than is helpful. And the emotions of the individual hearing the concern will make them more defensive, more impatient and less open to truly hear the concern than would be hoped. Just as "rehearsal" and peer coaching is a useful strategy to develop the muscle for voicing values effectively, so too these can be useful approaches to building the patience, selfmanagement and communication "scripts" for hearing these messages in ways that signal true receptivity.

There is no magic formula for listening for values just as there is no universal answer to the question of how to voice them. Circumstances and relationships will dictate different approaches. But the key lesson here is that we cannot express our values effectively if we do not listen to what matters to the persons we are trying to influence; and we cannot expect our employees to express their values-driven concerns if we typically "kill the messenger." And in the end, one of the best ways to build the muscle and the skill and the confidence to accomplish both of these goals is through practice and an intentional attention to sharing what has worked in the past.

\section{Questions to ask Ourselves as we Listen for Values}

- Consider at time when you expressed/acted on your values in the workplace: how much time did you spend listening to and trying to understand the perspectives and concerns of your audiences, as compared to the time spent preparing what you were going to say and do yourself?

- When was the last time you expressed/acted on your values in the workplace? Do you believe your audiences truly listened to you? Why or why not? If not, could you have done 
anything to encourage greater listening? And did you truly listen to what they were concerned about?

- When was the last time someone tried to express a values-based concern to you in the workplace? Were you listening? Why or why not?

- What can we do to make it easier, more natural to truly listen to the values-based concerns of our colleagues? When/why is it challenging? How can we become more comfortable listening?

- When a colleague expresses their concerns to us, how can we diminish the defensiveness that sometimes blocks us from truly hearing what the underlying issue is? Can GVV-style "rehearsal" help with this? One of the seven "pillars" of GVV is "Normalization". That is, recognizing that voicing and listening to values issues is a very predictable and inevitable part of any organizational practice. So through rehearsal we can become more comfortable, less emotional, more calm and clear.

- What organizational practices will we put into place to support more effective and consistent expression and listening to values-based concerns? 